\title{
A IDENTIFICAÇÃO DA ANSIEDADE POR MEIO DA ANÁLISE DA ÍRIS: uma possibilidade ${ }^{a}$
}

\author{
Léia F ortes SA L LES ${ }^{b}$, M aria Júlia Paes da SI LVAc
}

\section{RESUMO}

A ansiedade é um comportamento relacionado com doenças psicossomáticas. Por meio da avaliação da íris é possível determinar características de personalidade e intervir precocemente para evitar que algum comportamento mal adaptativo colabore para o desenvolvimento de uma doença. Os anéis de tensão sugerem predisposição para ansiedade. 0 objetivo deste trabal ho foi verificar a correlação dos anéis de tensão presentes na íris com o Inventário de Diagnóstico da Ansiedade T raço-Estado (IDATE). A pós aprovação pelo Comitê de Ética e Pesquisa, o estudo foi realizado com 62 funcionários do Centro de A perfeiçoamento em Ciências da Saúde da F undação Zerbini entre maio e julho de 2010. 0 coeficiente de correlação de Spearman mostrou correlação positiva e significativa entre o escore do ID AT E e a quantidade dos anéis de tensão, sugerindo que a presença destes sinais indicam maior predisposição para ansiedade. Com a identificação precoce deste comportamento temos chances de diminuir 0 desenvolvimento de doenças.

D escritores: Ansiedade. I ridologia. Prevenção de doenças. M edicina integrativa.

\section{RESUMEN}

L a ansiedad es uno de los comportamientos asociados a las enfermedades psicosomáticas. A través de la evaluación del iris es posi ble determinar los rasgos deper sonalidad y deintervención temprana para prevenir una mala conducta y evitar desar rollar una enfermedad. L os anillos de tensión sugieren una predisposición a la ansiedad. E I objetivo de este estudio fue eval uar la correlación de los anillos de estrés presentes en el iris con el Inventario D iagnóstico de Ansiedad Rasgo-E stado (IDATE). D espués dela aprobación por el Colegio deÉ tica el nvestigación, la encuesta sellevó a cabo con 62 funcionarios del Centro para el Avance de las Ciencias de la Salud F undación Z erbini entremayo y julio de2010. E I coeficientedecorrelación de Spearman mostró una cor relación positiva y significativa entrela puntuación del IDAT E yla cantidad deanillos de tensión, lo quesugiere quela presencia de estos signos indica una mayor predisposición a un comportamiento ansioso. Con la identificación temprana de este compor tamiento se puede reducir el desarollo de las enfermedades.

D escriptores: Ansiedad. I ridología. P revención de enfermedades. M edicina integral. T ítulo: L a identificación de la ansiedad a través del análisis de iris: una posibilidad.

\section{ABST RACT}

Anxiety is a behavior related to psychosomatic diseases. Through an evaluation of the iris it is possible to determineper sonality traits and to intervene early to refrain any non-adaptive behavior from causing psychosomatic diseases. Contraction fur rows suggest a predisposition to anxiety. T his study aimed to verify the cor relation of contraction furrows in theiris with theStateT rait A nxi ety Inventory (STAI). T his study was approved by the R esearch E thics Committee of the U niversity Of São Paulo, $\mathrm{School}$ of $\mathrm{N}$ ursing, and performed with 62 teachers and employees at the Center of $\mathrm{H}$ ealth Science of the Z erbineF oundation (CeFACS), from M ay to J uly, 2010. The Spearman coefficient showed a positive and statistically significant correlation betw een the STAI score and the quantity of contraction fur rows. This suggests that these signs indicate a predisposition to anxiety. W ith the early identification of this behavior we have chances to reduce the development of the disease.

Descriptors: Anxiety. I ridology. D isease prevention. Integrative medicine

$\boldsymbol{T}$ itle: $T$ heidentification of anxiety through the analysis of theiris: a possibility.

\footnotetext{
a D ados extraídos da monografia realizada em 2010 para o Curso de Especialização em T erapia Vibracional da E scola de Enfermagem na Universidade de São Paulo (EE-USP).

${ }^{b}$ E nfer meira E special ista em Iridologia e I risdiagnose, D outoranda pelo Prog rama de Pós-G raduação em Saúde do A dulto da E E-U SP, São Paulo, Brasil.

'Enfermeira, Professora T itular do D epartamento M édico-Cirúrgico da E E-USP, São Paulo, Brasil.
} 


\section{INT RODUÇÃO}

A ansiedade é um dos comportamentos mais relacionados com as doenças psicossomáticas. M étodos que minimizem este tipo de comportamento certamente ajudarão na prevenção de inúmeras doenças que tenham origem e/ ou agravamento nos fatores emocionais ${ }^{(1,2)}$. Por meio da análise da íris temos a possibilidade de localizar precocemente indivíduos ansiosos e orientá-los na mudança de padrão e hábitos de vida. A análise da íris tem se mostrado um instrumento valioso no conhecimento físico e emocional das pessoas. $\mathrm{Na}$ iridologia, a presença de anéis de tensão na íris sugere indivíduos ansiosos. T anto no Brasil, como no mundo, existem poucos estudos publicados sobre ansiedade e iridologia.

É pertinente e admissível, portanto, comparar os anéis de tensão com o já validado e aceito instrumento para medir ansiedade, o I nventáriode Diagnóstico da A nsiedade T raço-E stado (IDAT E $)^{(1,3)}$.

Há cerca de 2 mil anos atrás o filosofo E pictetus (50-130 a.C) declarou que "os homens não são perturbados pelas coisas, mas pela visão que têm das coisas".

A avaliação subjetiva de uma situação e as características de personalidade de cada pessoa modulam suas respostas a um evento. Os processos cognitivos de avaliação direcionam as reações aos fatores externos e refletem a relação única e mutável que existe entre o homem, seus valores, suas percepções e crenças, seus compromissos, pensamentos e seu ambiente ${ }^{(4)}$.

U m estímulo interno ou externo interpretado como perigoso ou ameaçador, desencadeará uma reação emocional caracterizada como um estado de ansiedade, que gera sensações subjetivas que se manifestam no organismo. É uma reação frente a uma ameaça, real ou imaginária. U ma forma de expressão do medo(5).

A ansiedade transparece por alterações fisiológicas como tremores, pal pitações e vertigens. Podem ainda aparecer hiperventilação, náuseas, diarréia, boca seca, insônia, fraqueza e inapetência. As alterações comportamentais incluem preocupação, inquietação, nervosismo, tensão e apreensão e podem aparecer sem que a presença de uma ameaça real seja identificada, podendo parecer aos demais como desproporcional à intensidade da emoção(6).

A ansiedade inconsciente costuma ser causa de diversas doenças e, quando trabal hada a nível do consciente, muitas doenças podem ser evitadas(2). Alguns estudos mostram a relação de distúrbios emocionais, como depressão eansiedade, no desenvolvimento e na manutenção da dor e de outros sintomas ${ }^{(7,8)}$. Outros discutem o ônus econômico e social resul tante da combinação ansiedade e depressão e sintomas fisicos de dor ${ }^{(9,10)}$.

O comportamento ansioso gera muitos problemas, detectar precocemente grupo de risco para esse comportamento e ensinar novas possibilidades de reação frente ao estresse, reduz o sofrimento humano(1).

I ridologia significa o estudo da íris e Í risdiagnose é uma ciência que permite identificar por meio da íris aspectos físicos, emocionais e mentais do indivíduo. É um método propedêutico que permite, através da observação da íris, conhecer num dado momento, a constituição geral e parcial do indivíduo, bem como os estágios evolutivos, agudo, subagudo, crônico e degenerativo das alterações que acometem um ou mais órgãos ou o organismo como um todo(11).

F undamentando a I ridologia, temos que a íris é uma extensão do cérebro, fartamente dotada de ter minais nervosos, minúsculos capilares sanguíneos eoutros tipos de tecidos especializados. Conectada com todos os órgãos e tecidos do corpo, via tál amo óptico e sistema nervoso, a íris tor na-se uma espécie de "tela de tel evisão" em miniatura que revela a condição das áreas mais remotas do organismo, por meio das mudanças do refluxo neurológico no estroma e nas fibras da íris ${ }^{(12)}$.

Os anéis de tensão ou anéis nervosos são círculos concêntricos completos ou parciais evidenciados na íris. A parecem como sulcos deprimidos com maior frequência na zona ciliar ${ }^{(11)}$ (F igura 1 ).

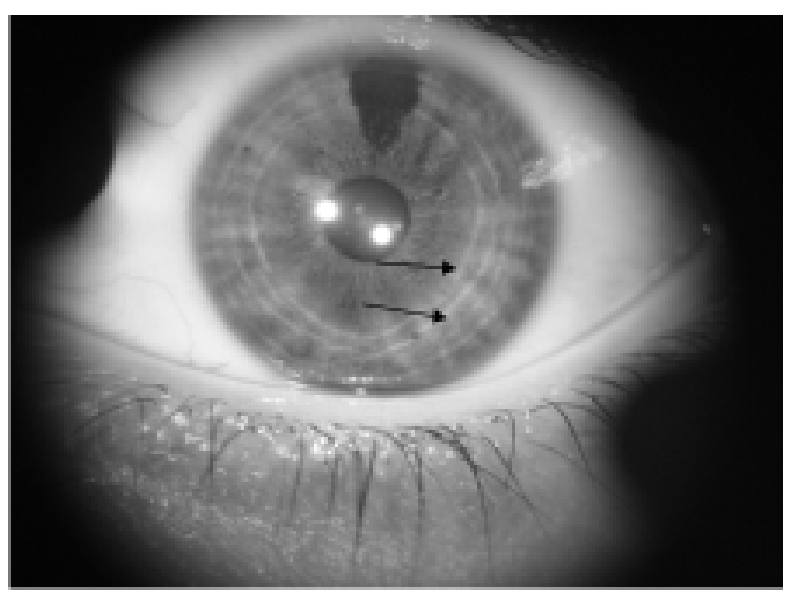

Figura 1 - Írís com anéis de tensão(1). São Paulo, 2010. 
A íris pode ou não ter anel de tensão. Quando presentes, variam em número de um a seis ${ }^{(11)}$.

Os anéis de tensão são verdadeiras rugas nas fibras das íris que se acumulam e aprofundam com 0 aumento do estresse. Estudos recentes indicam que há 60 anos, três anéis de tensão significariam um esgotamento nervoso iminente e, atualmente, esta mesma quantidade é média, sendo que muitas crianças já nascem com 4 a 5 anéis ${ }^{(12)}$.

Os anéis de tensão ainda indicam mau estado neurovascular, resultante da deficiência do suprimento nervoso e vascular, além de tônus muscular el evado com predisposição a dores, câimbras, cólicas e convulsões. Sugerem ainda tensão psíquica, impaciência, ansiedade, estresse, insônia e nervosismo(11-13).

Se por um lado, os indivíduos que possuem os anéis de tensão estão predispostos a uma série de comprometimentos orgânicos e psíquicos, por outro, são pessoas realizadoras que tentam al cançar seus objetivos. Para que não venham a desenvolver problemas orgânicos e nem psíquicos e se favoreçam deste perfil realizador, o importante é chegar a um equilíbrio. Os indivíduos com anéis de tensão podem encontrar o equilíbrio em atividades como al ongamentos, exercícios, relaxamento e meditação(11-13).

E m um estudo realizado em São Paulo com 346 indivíduos, a ansiedade apareceu $120 \%$ a mais no grupo que apresentava anéis de tensão na íris em relação ao grupo que não apresentava o sinal|(14). E m outro trabalho dos mesmos autores, apresentado no II Congresso de Investigação em Enfermagem I bero-A mericano e de Países de Língua Oficial Portuguesa de 2009, houve correlação significante entre a quantidade de anéis de tensão na íris e os escores do I nventário de Diagnóstico da Ansiedade T raço-E stado que mede ansiedade, sugerindo que estes sinais (anéis de tensão) podem ajudar na pré-diagnose de comportamentos ansiosos já na infância ${ }^{(15)}$. Por não terem sido encontrados outros estudos semel hantes, nesta pesquisa nos propomos a continuar a investigação da correlação entre anéis de tensão e o IDATE.

Dentre as profissões, a Enfermagem foi pioneira no reconhecimento das terapias complementares. A Resolução do Conselho Federal de Enfermagem (COFE N) 197/ 1997 estabelece e reconhece as T erapias A Iternativas como especialidade e/ ou qualificação do profissional de Enfermagem ${ }^{(16)}$.

Como a I ridologia permite conhecer por meio da íris aspectos físicos, emocionais e mentais do indivíduo, e tem como principal objetivo detectar distúrbios em evolução para precocemente intervir, a fim de que tal distúrbio não evolua para a doença, ela pode e deve ser utilizada para que a assistência de enfermagem ao paciente seja a mais completa possivel.

Basicamente as informações fornecidas pela íris podem ser utilizadas durante todo processo de enfermagem e também para facilitar a comunicação interpessoal. Para uma enfer meira especial ista em iridologia, a obser vação e análise da íris devem fazer parte do exame físico e os resultados devem nortear o processo de enfermagem e encaminhamento para outros profissionais, sempre que necessário(17).

A s informações captadas através da observação e da análise da íris podem ser utilizadas para direcionar e complementar a anamnese e o exame físico, fornecer novas pistas para a elucidação do diagnóstico, revelar outros sinais comprometedores e órgãos doentes e identificar determinados comportamentos para facilitar o relacionamento da equipe de enfermagem com o paciente ${ }^{(17)}$.

0 objetivo geral desta pesquisa foi comparar os resultados do I nventário de D iagnóstico da Ansiedade T raço-E stado com a presença dos anéis de tensão da íris. E, os específicos foram tipificar os entrevistados quanto à classificação da ansiedade, mensurada pel o I nventário de Diagnóstico da Ansiedade T raço-E stado e a anal isar a íris (quantidade e características dos anéis de tensão).

\section{MÉTODO}

T rata-se de um estudo exploratório, descritivo, de campo, transversal, com abordagem quantitativa ${ }^{(18)}$. A pesquisa foi realizada com docentes e funcionários do Centro de A perfeiçoamento em Ciências da Saúde da F undação Zerbini (CeFACS) no período de maio a julho de 2010, após aprovação do Comitê de Ética e Pesquisa da Escola de Enfermagem da U niversidade de São Paulo (Processo $n=889 / 2010 /$ CEP-E EU SP).

Com base na população de 120 funcionários e utilizando uma confiança de $80 \%$ e um erro de $5 \%$, chegou-se a um tamanho amostral de 58 funcionários $^{(19)}$. A amostra foi de conveniência, constituídas por indivíduos que desejaram participar do estudo dentro do período em que a pesquisadora estava na instituição.

Participaram do estudo 62 indivíduos após concordância e assinatura do Termo de Consentimento Livre e E sclarecido. 
Os dados foram coletados em uma sala cedida pela coordenação da instituição, onde os indivíduos foram atendidos individual mente. 0 preenchimento da ficha clínica e fotografia da íris foram realizados pela autora enfermeira especialista em I ridologia. A captação das imagens das íris direita e esquerda foi feita com uma câmara digital acoplada a uma lente iridológica com luminosidade adequada. As fotos foram gravadas em memory card.

A ansiedade foi avaliada pelo Inventário de Diagnóstico da Ansiedade T raço-E stado (IDATE), que é composto de duas escalas para medir dois conceitos distintos de ansiedade: Estado de ansiedade (condição cognitivo-afetiva transitória) e T raço de ansiedade (característica da personalidade). O T raço de ansiedade representa dados da personalidade do indivíduo e seus escores são menos sensíveis a mudanças decorrentes de situações ambientais. O Estado de ansiedade refere-se ao estado emocional transitório e os escores de ansiedadeestado podem variar em intensidade de acordo com situações do ambiente e flutuar no tempo. E m geral, caracteriza-se por sentimentos desagradáveis de tensão e apreensão, conscientemente percebidos, e por aumento na atividade do sistema nervoso autônomo. N este estudo foi utilizado o IDAT E traço. A classificação dos níveis de ansiedade é feita de acordo o escore obtido: baixo (20 a 34), moderado (35 a 49), el evado (50 a 64) e altíssimo (65 a $80)^{(20)}$. 0 inventário conta com 20 questões com respostas de múltipla escolha (escala tipo Likert) em que 0 individuo tem que preencher de forma natural sem se deter muito tempo em cada pergunta, conforme instruções que constam no instrumento.

Para efeito de comparação e respeitando literatura especializada no assunto ${ }^{(11-14)}$, os anéis de tensão foram quantificados e os indivíduos classificados com relação ao grau de ansiedade em: baixo (0 a 1), moderado (2 a 3), el evado (4 a 5) ealtíssimo (6 ou mais).

Para todos os entrevistados foi feita a seguinte pergunta: "Você se considera ansioso?", para saber se a auto percepção de sua ansiedade teria correlação com o resultado do inventário e/ ou com a presença dos anéis de tensão na íris.

Os dados foram tratados pela anál ise estatística, e os testes utilizados empregaram o coeficiente de corr elação de Spearman e o T este Exato de Fisher. 0 nível de significância adotado foi de $5 \%$. As estatísticas com $p$ descritivo < que 0,05 foram consideradas significativas.

\section{RESULTADOS}

Os 62 integrantes da pesquisa er am na maioria do sexo feminino $(88,7 \%)$, com idades entre 22 a 60 anos. A média de idade foi de 41 anos.

Todos os participantes apresentaram anéis de tensão (100\%). A penas 5 participantes (8\%) tinham 1 anel de tensão e a classificação de baixa ansiedade. 0 s outros apresentaram entre 2 e 5 anéis de tensão, classificando-se a maioria em ansiedade moderada (60\%), seguida de ansiedade elevada (32\%). N enhum dos participantes apresentou seis ou mais anéis de tensão na íris, o que é considerado altíssima ansiedade.

Quanto a profundidade dos sinais, 46 indivíduos $(74,2 \%)$ apresentaram anéis de tensão profundos e $16(25,8 \%)$ superficiais. T ambém $46(74,2 \%)$ entrevistados percebem-se ansiosos e $16(25,8 \%)$ não.

A pós análise estatística foi contastada que 0 instrumento de avaliação da ansiedade teve uma confiabilidade de $86 \%$ para esta amostra (Cronbach's A I pha = ,864).

H ouve correspondência entre o escore e classificação do IDATE com número de anéis de tensão da íris de 53 indivíduos (85\%). E aplicando-seo coeficiente de correlação não paramétrica de Spearman foi encontrado uma correlação positiva e significativa entre o escore do IDATE com a quantidade dos anéis de tensão (T abela 1).

T abela 1 - Correlação do IDAT E com quantidade de anéis de tensão. São Paulo, 2010.

\begin{tabular}{lcc}
\hline $\begin{array}{l}\text { Coeficiente de correlação } \\
\text { de postos de Spearmans }\end{array}$ & $\begin{array}{c}\text { Anéis de } \\
\text { tensão }\end{array}$ & E score \\
\hline $\begin{array}{l}\text { Anéis de tensão } \\
\text { Coeficiente de correl ação }\end{array}$ & 1,000 &, $698^{*}$ \\
Significância & 6 &, 000 \\
N & 62 & 62 \\
E score &, $698 *$ & 1,000 \\
$\quad$ Coeficiente de correl ação &, 000 & \\
Significância & 62 & 62 \\
N & & \\
\hline
\end{tabular}

* Correlação é significante ao nivel de 0,01.

Quanto à relação entre a profundidade dos anéis de tensão e a auto-percepção, o teste exato de F isher aponta associação significativa entre as duas variáveis, ou seja, indivíduos com anéis detensão profundos têm maior percepção do seu comportamento ansioso em relação à indivíduos que apresentam estes sinais superficiais. 


\section{DISCUSSÃO}

Estes resultados reafirmam os achados dos estudos anteriores de que os anéis de tensão sugerem ansiedade $\mathrm{e}^{(14,15)}$ e que há cor respondência entre esses sinais e a classificação de ansiedade medida pelo IDATE.

E $m$ 2007, pesquisadores suíços, que não trabal ham com iridologia, encontraram indícios de que a íris de seres humanos tem relação com o tipo de personalidade. A equipe da U niversidade de Ö rebro, da Suíça, analisou o "desenho" das íris de 428 pessoas comparando diferentes padrões de íris com os traços de per sonal idade dos indivíduos. Os pesquisadores descobriram que duas características da íris variam de acordo com a per sonalidade: a densidade das fibras e a existência de "sulcos" que se formam ao redor da pupila ${ }^{(21)}$, que na iridologia recebem o nome de anéis de tensão. É muito interessante ver que difer entes segmentos estão chegando a resultados semel hantes.

Os estados emocionais podem funcionar como gatilho para o surgimento de diversas doenças e devem ser identificados e tratados precocemente. Em um contexno de rápido envelhecimento da população mundial e aumento das doenças crônicas degener ativas, técnicas acessíveis, como a iridologia, que ajudam a reconhecer estes estados são bem vindas.

\section{CONCLUSÕES}

A maioria dos integrantes da pesquisa era do sexo feminino (88,7\%), com idade média de 41 anos.

Todos os participantes apresentaram anéis detensão (100\%) e a classificação da ansiedade, medida pela quantidade do sinal, foi baixa (8\%), moderada (60\%) e elevada (32\%). N enhum dos participantes apresentou ansiedade altíssima.

Os resultados apontam para uma correlação de $85 \%$ entre a classificação de ansiedade medida pelo I nventário de D iagnóstico da A nsiedade T raço-E stado e a quantidade dos anéis de tensão na íris. 0 coeficiente de correlação não paramétrica de Spearman demonstra correlação positiva e significativa entre o escore do IDATE com a quantidade dos anéis de tensão.

0 teste exato de Fisher sinaliza associação significativa entre a profundidade dos anéis de tensão e a auto percepção da ansiedade, ou seja, indivíduos com anéis de tensão profundos têm maior percepção do seu comportamento ansioso em relação à indivíduos que apresentam estes sinais superficiais.

Os iridologistas afirmam que os anéis de tensão sugerem predisposição para o comportamento ansioso e por meio deste estudo confirmamos, mais uma vez, a correlação destes sinais com a ansiedade e com o IDATE, instrumento já validado e reconhecido pela sociedade científica brasileira e inter nacional para medir ansiedade ${ }^{(3)}$.

Reconhecer precocemente o modo pelo qual 0 indivíduo reage frente ao estresse possibilita ensiná-lo novas formas de reação e, assim, diminuir a chance deste comportamento contribuir para a manifestação de uma doença.

A análise da íris vem se mostrando um instrumento seguro para somar aos meios de diagnósticos conhecidos. E la propicia um mapeamento físico e emocional do indivíduo, al ém de ser rápida, barata e e sem efeitos colaterais.

As práticas complementares, dentre elas a iridologia, devem ser utilizadas para que a assistência ao paciente seja mais completa possível. É necessário o investimento em pesquisas nesta área, que demonstra ser um campo promissor. As discussões e debates das práticas trazem um novo desafio para praticantes, profissionais e estudiosos: encontrar evidências de segur ança e efetividade das Ter apias A Iter nativas e Complementares por meio de model os de pesquisa adaptados à realidade própria dessas práticas.

\section{REFERÊ NCIAS}

1 Salles LF. E feito das essências florais em indivíduos ansiosos e com anéis de tensão na íris [ monografia] . São Paulo: Escola de Enfermagem, U niversidade de São Paulo; 2010.

2 M ay R. 0 significado da ansiedade: as causas da integração e desintegração da personalidade. Rio de Janeiro: Zahar; 1977.

$3 \mathrm{G}$ orenstein $\mathrm{C}$, A ndrade L. Validation of a Portuguese version of the Beck Depression Inventory and The State-T rait Anxiety Inventory in Brazilian subjects. Braz J M ed Biol Res. 1996;29(4):453-7.

4 Pontieri F M , Bachion M M . Crenças de pacientes diabéticos acerca da terapia nutricional e sua influência na adesão ao tratamento. Ciênc Saúde Colet [ I nternet] . 2010 [ citado $2010 \mathrm{dez} 10$ ];15(1):151-60. D isponível em: http:/ / www.scielo.br/ pdf/ csc/ v15n1/ a 21v15n1.pdf. 
5 Sadock BJ, Sadock VA. Compêndio de psiquiatria: ciência do comportamento e psiquiatria clinica. 9a ed. Porto Alegre: Artmed; 2007.

6 Suriano M LF, M acedo GPOS, M ichel JLM, Barros ALBL. I dentificação das características definidoras de medo e ansiedade em pacientes programadas para cirurgia ginecológica. Acta Paul Enferm. 2009;22(n esp):928-34.

7 Romão APM S. 0 impacto da ansiedade e da depressão na qualidade de vida de mulheres com dor pél vica crônica [ dissertação] . Ribeirão Preto: Faculdade de $M$ edicina de Ribeirão Preto, U niver sidade de São Paulo; 2008.

8 Lima RTA . F atores associados à disfunção temperomandibular em população com ansiedade e depressão [ tese]. Bauru: Faculdade de Odontologia de Bauru, Universidade de São Paulo; 2009.

9 M enezes $G B$, Fontenelle LF, M ululo S, Versiani M . Resistência ao tratamento nos transtor nos de ansiedade: fobia social, transtorno de ansiedade generalizada e transtorno do pânico. Rev Bras Psiquiatr [ I nternet] . 2007 [ citado $2010 \mathrm{dez} 10$ ];29(Supl 2):S5560. Disponível em: http:// www.scielo.br/ pdf/ rbp/ v29s2/ a04v29s2.pdf.

$10 \mathrm{M}$ artins VA . P sicossomática e transtor nos de somatização: caracterização da demanda em hospital escola no período de 1996-2004 [ dissertação] . Ribeirão P reto: Escola de E nfermagem de Ribeirão P reto, U niversidade de São Paulo; 2007.

11 Batello CF. I ridologia e irisdiagnose: o que os olhos podem revelar. 3ạ ed. São Paulo: G round; 2009.

12 Kalsa G S. I ridologia integrada: a ciência e a arte da revelação do holograma humano. São Paulo: M andras; 2009.
13 Batello CF. Psicoiridologia Jung e M étodo Rayld. São Paulo: Cartex; 2009.

14 Salles LF, Silva M JP. A incidência dos anéis de tensão na íris de pessoas que vivem em São Paulo. N ursing (São Paulo). 2005;81(8):86-90.

15 Salles LF, Silva M JP. Correlação entre ansiedade e anéis de tensão. Referência. 2011;3(3):7-11.

16 Conselho Federal de Enfermagem. Resolução COFE N-197/ 1997, de 19 de março de 1997: estabelece e reconhece as T erapias A Iter nativas como especialidade e/ ou qualificação do profissional de Enfermagem. In: Consel ho Regional de Enfermagem de São Paulo. D ocumentos básicos de enfermagem: enfermeiros, técnicos, auxiliares. São Paulo; 2001. p. $159-60$.

17 Salles LF. A prevalência de sinais iridológicos nos indivíduos com diabetes M ellitus [ dissertação] . São Paulo: E scola de E nfermagem, U niversidade de São Paulo; 2006.

18 Polit DF, Beck CT, Hungler BP. F undamentos da pesquisa em enfer magem: métodos, avaliação e utilização. Porto A legre: A rtmed; 2006.

19 D eter minação do tamanho de uma amostra [ Internet] . D isponível em: http:/ / www.fesppr.br/ centro pesq/Calculo_do_tamanho_da_amostra/ T amanho\% 20da\%20A mosträ\%20-\%201.pdf. [ A cessado em 12 Jan. 2010] .

20 Spielberger CD, G orsuch RL , L ushene RE . Inventário de ansiedade traço-estado - IDAT E. Rio de Janeiro: CEPA; 1979.

21 Larsson M, Pedersen NL, Stattin H. Associations between iris characteristics and personality in adulthood. Biol Psychol. 2007;75(2):165-75.

\section{Endereço da autora / Dirección del autor / Author's address:}

Léia Fortes Salles

Rua T ucuna, 742, ap. 132, Pompéia

05021-010, São Paulo, SP

E-mail: leia.salles@usp.br
Recebido em: 23/ 12/ 2010

A provado em: 01/ 02/ 2012 